\title{
Bach flows of product manifolds
}

\author{
Sanjit Das and Sayan Kar* \\ Department of Physics and Meteorology and Centre for Theoretical Studies \\ Indian Institute of Technology, Kharagpur, 721302, India
}

\begin{abstract}
We investigate various aspects of a geometric flow defined using the Bach tensor. Firstly, using a well-known split of the Bach tensor components for $(2,2)$ unwarped product manifolds, we solve the Bach flow equations for typical examples of product manifolds like $S^{2} \times S^{2}, R^{2} \times S^{2}$. In addition, we obtain the fixed point condition for general $(2,2)$ manifolds and solve it for a restricted case. Next, we consider warped manifolds. For Bach flows on a special class of asymmetrically warped four manifolds, we reduce the flow equations to a first order dynamical system, which is solved exactly to find the flow characteristics. We compare our results for Bach flow with those for Ricci flow and discuss the differences qualitatively. Finally, we conclude by mentioning possible directions for future work.
\end{abstract}

*Electronic address: sanjit@cts.iitkgp.ernet.in, sayan@iitkgp.ac.in 


\section{INTRODUCTION AND OVERVIEW}

The Bach tensor [1, 2] is a traceless, symmetric, conformally invariant second rank tensor in four dimensions which has the property of being divergence free. It is defined as:

$$
B_{i k}=\nabla^{j} \nabla^{l} C_{i j k l}+\frac{1}{2} R^{j l} C_{i j k l}
$$

Using the contracted second Bianchi identity $\left(\nabla^{l} R_{i j k l}=-\nabla_{i} R_{j k}+\nabla_{j} R_{i k}\right)$ and contracted Hessian of the Ricci tensor $\left(\nabla^{l} \nabla_{i} R_{l k}=\nabla_{i} \nabla^{l} R_{l k}+R_{i l} R_{k}^{l}-R_{i j k l} R^{j l}\right)$ we can write Eqn.(1) as

$$
B_{i k}=\square\left(R_{i k}-\frac{1}{6} R g_{i k}\right)-\frac{1}{3} \nabla_{i} \nabla_{k} R+\left(C_{i j k l}+R_{i j k l}-R_{i j} g_{k l}\right) R^{j l}
$$

where $C_{i j k l}$ is the Weyl tensor and $R_{i k}$ is the Ricci tensor. We mention that the above definitions are written using the Landau-Lifshitz sign convention [3] for the Riemann tensor (see [4] for a note on conventions and definitions). It is clear from the above definition, that the Bach tensor involves four spatial derivatives as well as squares of second derivatives of the metric tensor. It is, therefore a higher derivative and higher order geometric object.

In a recent article [5], it has been proposed that one may define a geometric flow (of metrics on a manifold) using the Bach tensor. The authors in [5] suggest a flow equation, following the equation for un-normalised Ricci flows [6, 8 - 10], given by

$$
\frac{\partial g_{i j}}{\partial t}=\mp \frac{\kappa^{2}}{2} B_{i j}
$$

where $t$ denotes the flow parameter (not the physical time).

The above flow can be shown as a gradient flow for the functional $\int_{\mathcal{M}}\left\|C_{i j k l}\right\|^{2} \mathrm{dm}$, where $\mathrm{dm}$ is a fixed measure, on the manifold $\mathcal{M} /$ Diff $\mathcal{M}$. This is similar to Ricci flow which is a gradient flow for the functional $\int_{\mathcal{M}} R \operatorname{dm}[7]$. The traceless property of the R. H. S. in the flow equation with the Bach tensor makes the above flow different from unnormalised Ricci flow-in fact there is no distinction between normalised and un-normalised flows defined using the Bach tensor. The short-time existence for higher order geometric flows (including Bach

flows) have been proved very recently in [11]. In our work here, we intend to understand the Bach flow of metrics through explicit, illustrative examples.

From a physics perspective, the Bach tensor arises in the well-known theory of conformal 
gravity (where instead of Einstein's equation one arrives at the Bach equation $B_{\mu \nu}=\kappa T_{\mu \nu}$ by varying the Weyl-squared action w.r.t the metric tensor). Conformal gravity has been extensively studied -for details see [12, 13]. On the other hand, solutions of the Bach flow equation also appear as solutions in the recently proposed five-dimensional Horava-Lifshitz theory of gravity. Let us quickly recall the discussion on this aspect as given in [5].

Consider five $(4+1)$ dimensional Horava-Lifshitz gravity. Assume a line element of the form:

$$
d s^{2}=-N^{2}(t) d t^{2}+g_{i j}\left(d x^{i}+N^{i} d t\right)\left(d x^{j}+N^{j} d t\right)
$$

where $N(t)$ and $N^{i}$ are the lapse and shift functions, as assumed in a standard ADM split of the line element. The indices $i, j$ run from 1 to 4 , since we are considering five spacetime dimensions. The action for Horava-Lifshitz gravity with anisotropic scaling $z=D=4$ is given as

$$
S_{H L}=\frac{2}{\kappa^{2}} \int d t d^{4} x \sqrt{g} N K_{i j} G^{i j k l} K_{k l}-\frac{\kappa^{2}}{8} \int d t d^{4} x \sqrt{g} N\left(\frac{1}{\sqrt{g}} \frac{\delta W}{\delta g_{i j}}\right) G_{i j k l}\left(\frac{1}{\sqrt{g}} \frac{\delta W}{\delta g_{k l}}\right)
$$

where $K_{i j}$ is the extrinsic curvature of the four dimensional Riemannian manifold with metric $g_{i j}$ and $G_{i j k l}$ is the de Witt metric in a superspace with parameter $\lambda$. We take $\lambda<\frac{1}{4}$ so that the Euclidean action is bounded below. $W[g]$ is specified using detailed balance and is given as

$$
\begin{array}{r}
W[g]=W_{w e y l}+W_{R^{2}}+W_{R}+W_{\Lambda_{W}} \\
=\int d^{4} x\left(a C_{i j k l} C^{i j k l}+b R^{2}-c R-2 c \Lambda_{W}\right)
\end{array}
$$

Solutions to $4+1$ Horava-Lifshitz gravity can thus be obtained from the geometric flow equation,

$$
\frac{1}{N(t)} \frac{\partial g_{i j}}{\partial t}= \pm \frac{\kappa^{2}}{2 \sqrt{g}} G_{i j k l} \frac{\delta W[g]}{\delta g_{k l}}+\nabla_{i} \xi_{j}+\nabla_{j} \xi_{i}
$$

Choosing $W[g]=W_{W e y l}$ and normalising the lapse to 1 , one gets the Bach flow equation quoted earlier. Thus, we note that the Bach flow has a physically relevant origin and is obtainable via an action principle.

The initial analysis in this article is based on a well-known result on the Bach tensor for 
the so-called $(2,2)$ product manifolds. The line element on such manifolds are given as:

$$
d s^{2}=g_{\mu \nu}^{(1)}\left(x^{\alpha}\right) d x^{\mu} d x^{\nu}+g_{a b}^{(2)}\left(x^{c}\right) d x^{a} d x^{b}
$$

where $x^{\mu}(\mu=1,2)$ and $x^{a}(a=3,4)$ are the coordinates on manifolds of dimension two. $g_{\mu \nu}$ and $g_{a b}$ depend only on $x^{\mu}$ and $x^{a}$, respectively. Such a construction is known as an unwarped product manifold- the unwarped property following from the fact that the metric tensor on the individual, two dimensional manifolds are dependent on the coordinates on each, respective manifold, i.e. $g_{\mu \nu}^{(1)}\left(x^{\alpha}\right)$ does not depend on $x^{c}$ and $g_{a b}^{(2)}\left(x^{c}\right)$ does not depend on $x^{\alpha}$. For such line elements one can check [14, 15] that the Bach tensor splits as follows:

$$
\begin{gathered}
B_{\mu \nu}=\frac{1}{3} \nabla_{\mu} \nabla_{\nu}{ }^{(1)} R-\frac{1}{3} g_{\mu \nu}^{(1)}\left\{\nabla^{\alpha} \nabla_{\alpha}{ }^{(1)} R-\frac{1}{2} \nabla^{a} \nabla_{a}{ }^{(2)} R+\frac{1}{4}\left({ }^{(1)} R^{2}-{ }^{(2)} R^{2}\right)\right\} \\
B_{a b}=\frac{1}{3} \nabla_{a} \nabla_{b}{ }^{(2)} R-\frac{1}{3} g_{a b}^{(2)}\left\{\nabla^{a} \nabla_{a}{ }^{(2)} R-\frac{1}{2} \nabla^{\mu} \nabla_{\mu}{ }^{(1)} R+\frac{1}{4}\left({ }^{(2)} R^{2}-{ }^{(1)} R^{2}\right)\right\}
\end{gathered}
$$

Hence, the Bach tensor is exclusively determined in terms of the derivatives of the Ricci scalars of the individual two dimensional manifolds in the product.

Our article is organised as follows. In Section II we analyse Bach flows on simple, unwarped product manifolds using the above mentioned decomposition. Further, in Section III, we obtain the fixed point equation of Bach flows on unwarped product manifolds and find some analytical, as well as numerical solutions. In Section IV, we investigate what might happen if we considered warped spacetimes, in paricular four dimensional Lorentzian spacetimes which are asymmetrically warped. It turns out that for a specific family of warped spacetimes, the flow equations reduce to a dynamical system which can be analytically solved. Finally, in Section V, we conclude with a summary and some perspectives.

\section{BACH FLOWS ON $S^{2} \times S^{2}$ AND $S^{2} \times R^{2}$}

The simplest example of unwarped $(2,2)$ product manifolds are $S^{2} \times S^{2}$ and $S^{2} \times R^{2}$. Let us discuss the $S^{2} \times S^{2}$ case first.

In order to discuss geometric flows, we assume that the line element on the $S^{2} \times S^{2}$ 
product manifold is given as

$$
d s^{2}=A^{2}(t) d s_{(1)}^{2}+B^{2}(t) d s_{(2)}^{2}
$$

where $A^{2}(t)$ and $B^{2}(t)$ are the scale factors, dependent on the flow parameter $t$, and $d s_{(1,2)}^{2}$ are the line elements on the round two-sphere of unit radius.

The Bach flow equations, using the split mentioned in the Introduction, turn out to be

$$
\begin{aligned}
& \frac{d f}{d t}= \pm \frac{\kappa^{2}}{24}\left(\frac{4}{f^{2}}-\frac{4}{g^{2}}\right) f \\
& \frac{d g}{d t}= \pm \frac{\kappa^{2}}{24}\left(\frac{4}{g^{2}}-\frac{4}{f^{2}}\right) g
\end{aligned}
$$

where $f(t)=A^{2}(t)$ and $g(t)=B^{2}(t)$.

It is easy to note (since the Bach tensor is traceless) that

$$
f(t) g(t)=A^{2}(t) B^{2}(t)=\text { Constant }
$$

i.e. $A^{2} B^{2}$ is conserved along the flow. This aspect clearly distinguishes the Bach flow from un-normalised Ricci flows. Secondly, the solutions for $f$ and $g$ (using the + sign in the above equations) are obtained as:

$$
\begin{aligned}
f^{2}(t) & =A^{4}(t)=C_{1} \tanh \left(\frac{2 t^{\prime}}{C_{1}}+C_{2}\right) \\
g^{2}(t) & =B^{4}(t)=C_{1} \operatorname{coth}\left(\frac{2 t^{\prime}}{C_{1}}+C_{2}\right)
\end{aligned}
$$

where $C_{1}$ and $C_{2}$ are constants and $t^{\prime}$ is the $t$ rescaled with $t^{\prime}=\frac{\kappa^{2}}{6} t$.

Thus, $A(t)$ has a past singularity (curvature diverges) but exists eternally in the future. On the other hand $B(t)$ is infinitely large at the $t$ value where $A(t)$ becomes zero, but, asymptotically, goes to the same constant as $A(t)$. The product $A^{4}(t) B^{4}(t)=C_{1}^{2}$.

We have numerically solved the Bach flow equations for some particular initial values of $A_{0}, B_{0}$ as shown in Fig.(1). Note that the generic features are quite different from Ricci flows of similar product manifolds, which we have discussed in one of our earlier works [16].

Let us now turn to $S^{2} \times R^{2}$. Here one of the Ricci scalars is zero, and the flow equations 


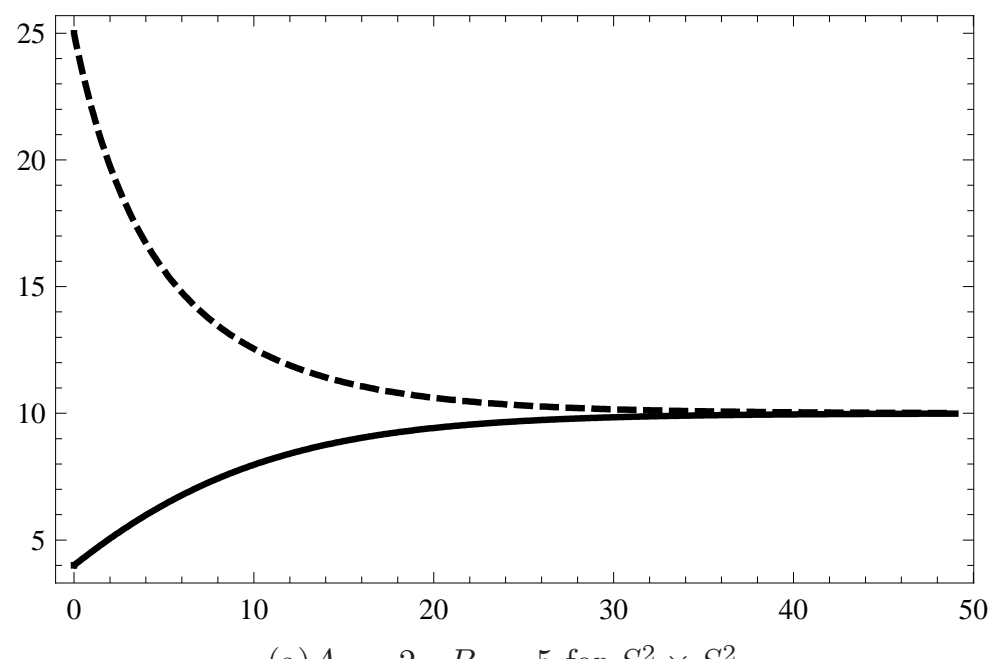

(a) $A_{0}=2, B_{0}=5$ for $S^{2} \times S^{2}$

FIG. 1: $A^{2}(t)$ (continuous) and $B^{2}(t)$ (dashed).The horizontal axis is $t, T_{s}=-6.35$.

are even more simple. We have

$$
\begin{gathered}
\frac{d f}{d t}= \pm \frac{1}{3} \frac{1}{f} \\
\frac{d g}{d t}=\mp \frac{1}{3} \frac{g}{f^{2}}
\end{gathered}
$$

The solutions for $f$ and $g$ are given as,

$$
\begin{aligned}
& f(t)=A^{2}(t)=\sqrt{t \mp \frac{3 C}{2 \kappa^{2}}} \\
& g(t)=B^{2}(t)=\frac{1}{\sqrt{t \mp \frac{3 C}{2 \kappa^{2}}}}
\end{aligned}
$$

where $C$ is a constant of integration and as before, $A^{2} B^{2}$ is conserved along the flow. Here too we note that while $A^{2}(t)$ is infinite at some $t, B^{2}(t)$ becomes zero there. Fig.(2) shows the numerical evolution of the scale factors for some particular initial values. Once again the results are very different from the standard Ricci flow evolution [16]

We may also consider products like $S^{2} \times H^{2}, H^{2} \times H^{2}$ and $R^{2} \times H^{2}$. However, there is no change in the results for the above-mentioned manifolds with constant curvature metrics on them. This is because the Bach tensor depends on the square of the Ricci scalar of the individual two manifolds-thus having negative curvature does not yield anything new. In other words, Bach flow on $(2,2)$ manifolds of constant curvature do not see the sign of the 


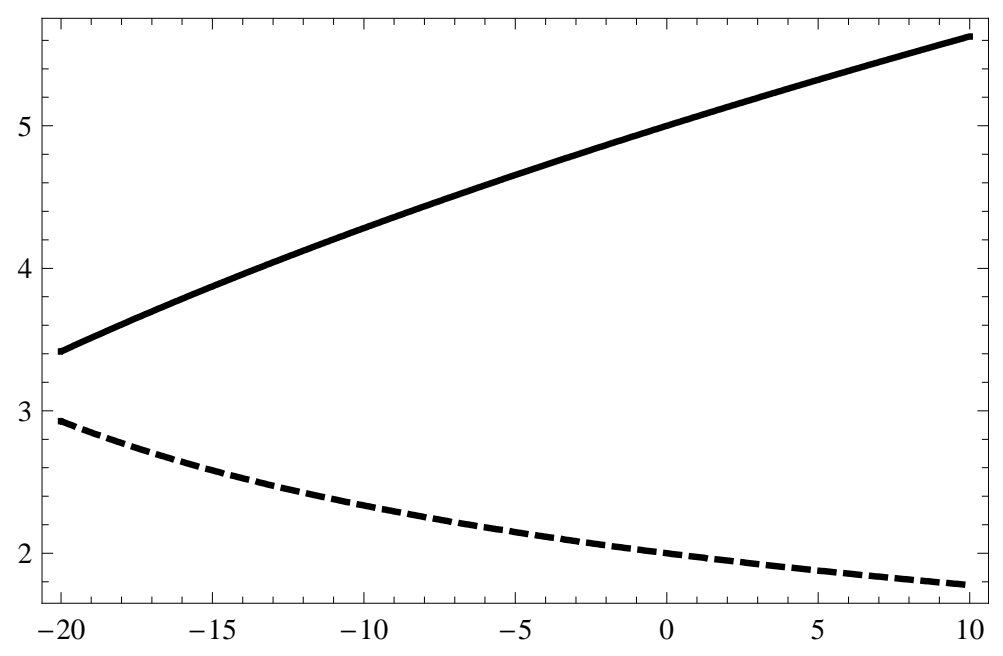

(a) $A_{0}=5, B_{0}=2, T_{s}=-37.5$ for $S^{2} \times R^{2}$

FIG. 2: $A^{2}(t)$ (continuous) and $B^{2}(t)$ (dashed). The horizontal axis is $t$.

Ricci scalar, of the individual two manifolds. This is another major point of difference with Ricci flows where differences arise if one considers products like $H^{2} \times H^{2}$ or $H^{2} \times R^{2}$ or $H^{2} \times S^{2}$ (see [16] for details). It is also worth noting that on an Einstein manifold the Bach tensor is zero. So for constant curvature four manifolds like $S^{4}$ and $H^{4}$ there is no Bach flow.

\section{FIXED POINTS IN BACH FLOW}

In this section we will find the fixed points of Bach flows on $\mathcal{M}^{2} \times \mathcal{M}^{2}$ manifolds. The fixed point equations are trivial and follow from the equations,

$$
B_{\mu \nu}=0 \quad ; \quad B_{a b}=0
$$

Taking the trace of the above equations w.r.t $g^{\mu \nu}$ and $g^{a b}$ and after some straightforward algebra, we find that the conditions for fixed points turn out to be

$$
\begin{aligned}
\nabla_{\mu} \nabla_{\nu}{ }^{(1)} R-\frac{1}{2} g_{\mu \nu} \nabla^{\alpha} \nabla_{\alpha}{ }^{(1)} R & =0 \\
\nabla_{a} \nabla_{b}{ }^{(2)} R-\frac{1}{2} g_{a b} \nabla^{c} \nabla_{c}{ }^{(2)} R & =0
\end{aligned}
$$


It may be mentioned here that, each of the above conditions can be recast as a conformal Killing equation for the conformal Killing vector $\nabla_{\nu}^{(1)} R$ or $\nabla_{a}^{(2)} R$ [17]. Thus, the Ricci scalars of the individual two dimensional manifolds must necessarily satisfy a constraint, in order to be fixed points. We will now explore these constraints in greater detail.

Let us assume the generic metric on the $(2,2)$ product manifold as

$$
d s^{2}=\Omega_{1}^{2}(x, y, t)\left(d x^{2}+d y^{2}\right)+\Omega_{2}^{2}\left(x^{\prime}, y^{\prime}, t\right)\left(d x^{\prime 2}+d y^{\prime 2}\right)
$$

i.e. each two-manifold, in the chosen coordinates, is conformally flat. Using the fixed point condition for either one of them, we obtain

$$
\nabla_{\mu} \nabla_{\nu}\left[e^{-\eta_{1}} \nabla_{E}^{2} \eta_{1}\right]-\frac{1}{2} \Omega_{1}^{2} \delta_{\mu \nu} \nabla^{\alpha} \nabla_{\alpha}\left[e^{-\eta_{1}} \nabla_{E}^{2} \eta_{1}\right]=0
$$

where $\eta_{1}=\ln \Omega_{1}^{2}$ and $\nabla_{E}^{2}$ is the Laplacian in Euclidean space $\left(\Omega_{(1,2)}^{2}=1\right)$. A similar equation exists for $\Omega_{2}^{2}$ as well.

From the fixed point condition one can also arrive at a pair of partial differential equations involving the Ricci scalar and the conformal factor. These are

$$
\begin{array}{r}
\frac{\partial^{2(1)} R}{\partial x^{2}}-\frac{\partial^{2(1)} R}{\partial y^{2}}-\frac{\partial \eta_{1}}{\partial x} \frac{\partial^{(1)} R}{\partial x}+\frac{\partial \eta_{1}}{\partial y} \frac{\partial^{(1)} R}{\partial y}=0 \\
\frac{\partial^{2(1)} R}{\partial x \partial y}-\frac{1}{2} \frac{\partial \eta_{1}}{\partial y} \frac{\partial^{(1)} R}{\partial x}-\frac{1}{2} \frac{\partial \eta_{1}}{\partial y} \frac{\partial^{(1)} R}{\partial x}=0
\end{array}
$$

Similar equations also exist for $\Omega_{2}^{2}$ (or $\eta_{2}$ and ${ }^{(2)} R$ ).

To find a simple solution, one can assume $\eta_{1}$ (or $\Omega_{1}$ ) to be a function of $x$ alone. In that case, the second equation is automatically satisfied. The first equation, on the other hand, gives,

$$
\frac{d^{2(1)} R}{d x^{2}}-\frac{d \eta_{1}}{d x} \frac{d^{(1)} R}{d x}=0
$$

Assuming

$$
\alpha=\frac{d^{(1)} R}{d x}
$$

we get,

$$
\alpha=C_{1} \Omega_{1}^{2}
$$


Substituting

$$
{ }^{(1)} R=-e^{-\eta_{1}} \frac{d^{2} \eta_{1}}{d x^{2}}
$$

we obtain the following equation for $\eta_{1}$.

$$
\eta_{1}^{\prime \prime \prime}-\eta_{1}^{\prime \prime} \eta_{1}^{\prime}=C_{2} e^{2 \eta_{1}}
$$

where the prime denotes differentiation w.r.t. $x$ and $C_{2}=-C_{1}$. We can find a solution with the following ansatz,

$$
\eta_{1}=\ln \Omega_{1}^{2}=\beta \ln x
$$

Substituting this back in the third-order differential equation, we obtain

$$
\frac{2 \beta}{x^{3}}+\frac{\beta^{2}}{x^{3}}=C_{2} x^{2 \beta}
$$

One can check that we have a consistent solution if

$$
\beta=-\frac{3}{2} \quad ; \quad C_{2}=2 \beta+\beta^{2}=-\frac{3}{4}
$$

Therefore, we have,

$$
\Omega_{1}^{2}=x^{\beta}=x^{-\frac{3}{2}}
$$

Similarly, assuming $\Omega_{2}^{2}$ as a function of $x^{\prime}$ alone we will end up with the same solution for $\Omega_{2}^{2}$ with $x$ replaced by $x^{\prime}$. The resulting line element is given as

$$
d s^{2}=\frac{1}{x \sqrt{x}}\left(d x^{2}+d y^{2}\right)+\frac{1}{x^{\prime} \sqrt{x^{\prime}}}\left(d x^{2}+d y^{\prime 2}\right)
$$

The Ricci scalar, R, of the four manifold, turns out to be

$$
R=-\frac{3}{2}\left(\frac{1}{\sqrt{x}}+\frac{1}{\sqrt{x^{\prime}}}\right)
$$

One can further convert the third order equation into a dynamical system by defining 


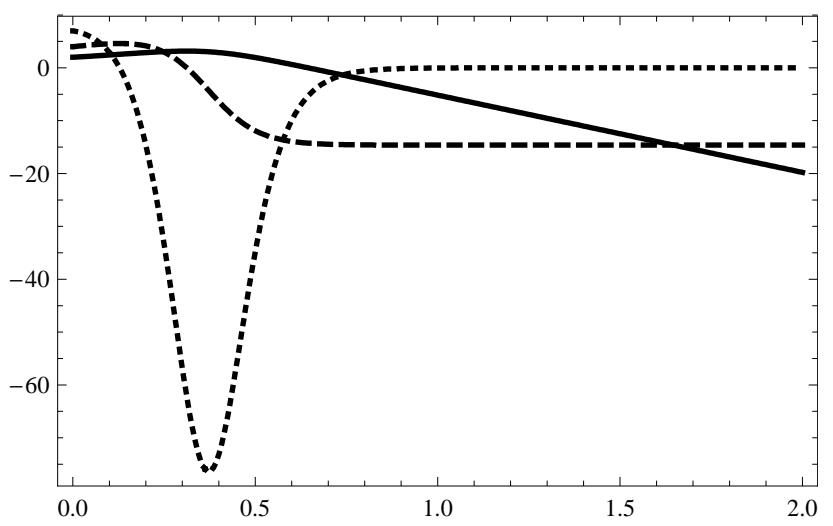

(a) $\eta(t)$ (continuous), $\xi(t)$ (dashed) and $\zeta(t)$ (dotted)

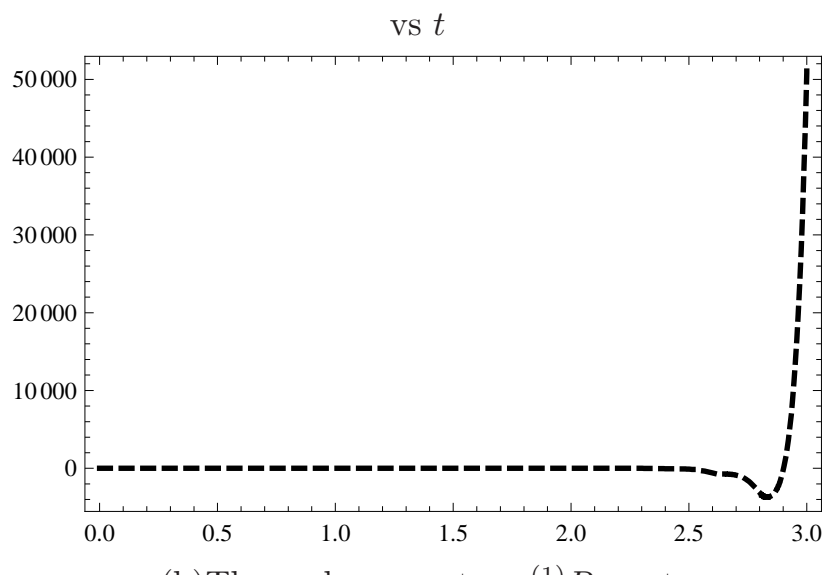

(b) The scalar curvature ${ }^{(1)} R$ vs. $\mathrm{t}$

FIG. 3: Fixed point analysis on $\mathcal{M}^{2} \times \mathcal{M}^{2}$ for the restricted case mentioned in the text.

$\eta_{1}^{\prime}=\eta^{\prime}=\xi\left(\right.$ we have renamed $\eta_{1}$ as $\left.\eta\right)$. We have

$$
\begin{array}{r}
\eta^{\prime}=\xi \\
\xi^{\prime}=\zeta \\
\zeta^{\prime}=\zeta \xi+C_{2} e^{2 \eta}
\end{array}
$$

This system can now be solved numerically for the general case to obtain desired results. We have taken initial values as $\eta_{0}=2, \xi_{0}=5, \zeta_{0}=7, C_{2}=-0.75$ and numerically solved the dynamical system. The results are shown in Fig.(3(a). Additionally, the variation of the scalar curvature ${ }^{(1)} R=-e^{-\eta} \zeta$ is also found from Eqn.(32) and is shown in Fig. (3(b). ${ }^{(1)} R$ diverges to positive infinity because $\zeta$ becomes zero (from the negative side) for large $t$ and $e^{-\eta}$ grows to very large positive values ( $\eta$ tends to large negative values for large $t$ ). 


\section{ASYMMETRICALLY WARPED PRODUCTS}

Let us now turn to a discussion on warped products. As a prototype family of warped product four manifolds let us assume the line element as

$$
d s^{2}=-A^{2}(t) e^{2 f(\sigma)} d \tau^{2}+B^{2}(t) e^{2 g(\sigma)}\left[d x^{2}+d y^{2}\right]+C^{2}(t) d \sigma^{2}
$$

Topologically the above manifold is $\mathbb{R}^{3} \times \mathbb{R}$ or $\mathbb{R}^{3} \times \mathbb{S}^{1}$ or $\mathbb{R}^{3} \times \mathbb{S}^{1} / \mathbb{Z}_{2}$ depending on the domain of the coordinate $\sigma$. The warping introduces curvature properties. The above line element is largely motivated by the so-called brane--world models with asymmetric warping [18], though with one dimension less. Note that $f \neq g$ is necessary in order to have a non-zero Bach tensor. To keep things simple let us assume further

$$
f(\sigma)=k_{1} \sigma \quad ; \quad g(\sigma)=k_{2} \sigma
$$

We now find the Bach tensor and, subsequently the Bach flow equations for the $A^{2}, B^{2}$ and $C^{2}$.

The Bach tensor components for the abovementioned line element are,

$$
\begin{aligned}
B_{t t}= & -\frac{A^{2}}{3 C^{4}} \alpha e^{2 k_{1} \sigma} \\
B_{x x}=B_{y y}= & -\frac{B^{2}}{3 C^{4}} \beta e^{2 k_{2} \sigma} \\
& B_{z z}=\frac{1}{3 C^{2}} \gamma
\end{aligned}
$$

where $\alpha, \beta$ and $\gamma$ are given as,

$$
\begin{gathered}
\alpha=k_{1}\left(k_{1}-k_{2}\right)\left(k_{1}-4 k_{2}\right)\left(k_{1}+3 k_{2}\right) \\
\beta=k_{1}\left(k_{1}-k_{2}\right)\left(k_{1}-4 k_{2}\right)\left(k_{1}+k_{2}\right) \\
\gamma=k_{1}\left(k_{1}-k_{2}\right)\left(k_{1}-4 k_{2}\right)\left(k_{1}-k_{2}\right)
\end{gathered}
$$

It is easy to check that the above components satisfy the traceless and divergence free 
condition on the Bach tensor. Tracelessness of Bach tensor gives contraint on $\alpha, \beta$ and $\gamma$ as

$$
\alpha-2 \beta+\gamma=0
$$

We can further verify that it is divergence free $\left(\nabla^{i} B_{i k}=0\right)$. All the components of $\nabla^{i} B_{i k}$ trivially go to zero expect for $k=3$. Therefore, we have only one non-trivial expression given as

$$
\nabla^{i} B_{i \sigma}=-\frac{2}{3 C^{4}}\left[k_{1}(\alpha-\beta)+k_{2}(\alpha-3 \beta)\right]
$$

which is essentially zero after substituting the values of $\alpha$ and $\beta$.

We also note that the Bach tensor is zero when $k_{1}=k_{2}$ and when $k_{1}=0$. The former is a trivial conformally flat case. For the latter, i.e. with $k_{1}=0$, conformal flatness is not directly visible. However, we can see it as follows (assume that $A, B$ and $C$ are scaled into the coordinates).

$$
\begin{array}{r}
d s^{2}=-d t^{2}+e^{2 k_{2} \sigma}\left(d x^{2}+d y^{2}\right)+d \sigma^{2} \\
=e^{2 k_{2} \sigma}\left[-e^{-2 k_{2} \sigma} d t^{2}+d x^{2}+d y^{2}+e^{-2 k_{2} \sigma} d \sigma^{2}\right] \\
=\frac{1}{k_{2} \sigma^{\prime 2}}\left[\sigma^{\prime 2} d t^{\prime 2}+d \sigma^{\prime 2}+d x^{2}+d y^{2}\right] \\
=\frac{1}{\eta^{2}-\xi^{2}}\left[-d \eta^{2}+d \xi^{2}+d \sigma^{\prime 2}+d x^{2}+d y^{2}\right]
\end{array}
$$

where $t^{\prime}=k_{2} t$ and, in the last step we have used a two dimensional Rindler like transformation from $\sigma^{\prime}, t^{\prime}$ to $\eta, \xi$. It is interesting to note that for $k_{1}=4 k_{2}$ all the components of Bach tensor are zero, i.e the metric is Bach flat. However, it is not conformally flat for $k_{1}=4 k_{2}$ because of the presence of non vanishing components of Weyl tensor. Thus, as is well-known, for metrics on manifolds of dimension $n \geq 4$ the vanishing of Weyl tensor components ensures that the metric is conformally flat (and Bach flat, for $n=4$ ) but a zero Bach tensor does not necessarily imply conformal flatness in four dimensions. 
Using the above, we can easily write down the Bach flow equations as,

$$
\begin{aligned}
& \frac{d A^{2}}{d t}=\mp \frac{\kappa^{2}}{2} \alpha \frac{A^{2}}{3 C^{4}} \\
& \frac{d B^{2}}{d t}= \pm \frac{\kappa^{2}}{2} \beta \frac{B^{2}}{3 C^{4}} \\
& \frac{d C^{2}}{d t}=\mp \frac{\kappa^{2}}{2} \gamma \frac{1}{3 C^{2}}
\end{aligned}
$$

We can absorb $\frac{\kappa^{2}}{6}$ by rescaling $t$ and easily obtain the exact solutions for the dynamical system. Here we have two sets of solutions depending on the sign used in the R. H. S. of the flow equations. Thus,

$$
\begin{aligned}
& C^{2}(t)=\left(2 C_{0}^{4}-2 \gamma t\right)^{1 / 2} \quad \text { for } t<\frac{C_{0}^{4}}{\gamma} \\
& B^{2}(t)=B_{0}^{2}\left(2 C_{0}^{4}-2 \gamma t\right)^{-\beta / 2 \gamma} \quad \text { for } t<\frac{C_{0}^{4}}{\gamma} \\
& A^{2}(t)=A_{0}^{2}\left(2 C_{0}^{4}-2 \gamma t\right)^{\alpha / 2 \gamma} \quad \text { for } t<\frac{C_{0}^{4}}{\gamma}
\end{aligned}
$$

For the backward flow, the solutions will be:

$$
\begin{gathered}
C^{2}(t)=\left(2 C_{0}^{4}+2 \gamma t\right)^{1 / 2} \\
B^{2}(t)=B_{0}^{2}\left(2 C_{0}^{4}+2 \gamma t\right)^{-\beta / 2 \gamma} \\
A^{2}(t)=A_{0}^{2}\left(2 C_{0}^{4}+2 \gamma t\right)^{\alpha / 2 \gamma}
\end{gathered}
$$

The expressions of $\alpha, \beta$ and $\gamma$ reveal that they vanish for $k_{1}=0, k_{1}=k_{2}$ or $k_{1}=4 k_{2}$. However, for non-vanishing $\gamma, \alpha$ or $\beta$ can still be zero if $k_{1}=-3 k_{2}$ or $k_{1}=-k_{2}$, respectively. These cases are quite easy to understand from the Bach flow equations. The solutions will be the same as quoted earlier except that either $A(t)=$ const. (for $\alpha=0$ ) or $B(t)=$ const. (for $\beta=0)$. We can choose values of $k_{1}$ and $k_{2}$ to explore the solutions of the evolution equations explicitly. We have accounted for two different regimes of $k_{1}$ and $k_{2}: k_{1}>4 k_{2}$ and $k_{1}<4 k_{2}$ respectively for forward and backward flows. Even $k_{1}<4 k_{2}$ has two sub-domains $-k_{1}<k_{2}$ and $k_{2}<k_{1}<4 k_{2}$. Below, we discuss each of the abovementioned cases in detail. In the corresponding figures, $A^{2}(t), B^{2}(t)$ and $C^{2}(t)$ are denoted by a bold line, a dashed line and 
a dotted line, respectively. The initial conditions are taken as $A_{0}^{2}=2, B_{0}^{2}=5$ and $C_{0}^{2}=7$. The left and right figures in Fig,4 are for forward and backward flows respectively.

A. $\alpha, \beta, \gamma \neq 0: \mathbf{k}_{1}>4 \mathbf{k}_{\mathbf{2}}$

In Fig $4(\mathrm{a})$ we have taken $k_{1}=8$ and $k_{2}=1$ for which $(\alpha, \beta, \gamma)=(2464,2016,1568)$. In this regime of $k_{1}$ and $k_{2}$ both $A^{2}(t)$ and $C^{2}(t)$ are decreasing whereas $B^{2}(t)$ is increasing. Thus, it is an ancient solution and develops a future singularity at $T_{s}=0.016$. Contrarily, the backward flow emerges as an immortal kind of flow with a past singularity at $T_{s}=-0.016$. Here $B^{2}(t)$ is decreasing while $A^{2}(t)$ and $C^{2}(t)$ increase and eventually diverge after crossing each other (checked, but not shown in figure) at some time in future. We also note that $B^{2}(t)$ and $A^{2}(t)$ cross each other and diverge for larger $t$.

B. $\alpha, \beta, \gamma \neq 0: \mathbf{k}_{\mathbf{1}}<\mathbf{4} \mathbf{k}_{\mathbf{2}}\left(k_{1}<k_{2}\right)$

Fig 4(b) shows a sub-regime of $k_{1}$ and $k_{2}$ where $k_{1}<k_{2}$. Here we choose $k_{1}=1$ and $k_{2}=5$ for which $(\alpha, \beta, \gamma)=(1216,456,-304)$. In the forward flow, both $B^{2}(t)$ and $C^{2}(t)$ deviate from each other after meeting at a point. $A^{2}(t)$ decays and asymptotically reaches a constant value. This is an immortal flow having a past singularity at $T_{s}=-0.081$. On the other hand, the backward flow is an ancient solution having a future singularity at $T_{s}=0.081$.

\section{C. $\alpha, \beta, \gamma \neq 0: \mathbf{k}_{\mathbf{1}}<4 \mathbf{k}_{\mathbf{2}}\left(k_{2}<k_{1}<4 k_{2}\right)$}

Fig $4(\mathrm{c})$ shows the other sub-regime of $k_{1}$ and $k_{2}$ where $k_{2}<k_{1}<4 k_{2}$. Here we choose $k_{1}=7$ and $k_{2}=3$ for which $(\alpha, \beta, \gamma)=(-2240,-1400,-560)$. In the forward flow, both $A^{2}(t)$ and $C^{2}(t)$ deviate from each other after crossing at some $t . B^{2}(t)$ crosses $C^{2}(t)$ and asymptotically reaches a constant value. $A^{2}(t)$, while increasing, crosses both $B^{2}(t)$ and $C^{2}(t)$ at two different $t$ values. It is an immortal flow having a past singularity at $T_{s}=-0.0437$. On the other hand, the backward flow is an ancient solution having a future singularity at $T_{s}=0.0437$. 

D. $\alpha=0, \beta, \gamma \neq 0: \mathbf{k}_{\mathbf{1}}<\mathbf{4} \mathbf{k}_{\mathbf{2}}\left(k_{1}<k_{2}\right)$

We will now focus on a special case where $\beta, \gamma$ are non zero but $\alpha$ is zero. The figure on the right in Fig 5 depicts this case. This is possible for $k_{1}=-3 k_{2}$ which lies in the regime of $k_{1}<k_{2}$. This is an ancient solution with future singularity at $T_{s}=0.073$. We can see that the vanishing of $\alpha$ flips the nature of the solution from immortal to ancient.

E. $\beta=0, \alpha, \gamma \neq 0: \mathbf{k}_{\mathbf{1}}<\mathbf{4} \mathbf{k}_{\mathbf{2}}\left(k_{1}<k_{2}\right)$

A similar feature appears for $\beta=0$ (i.e $k_{1}=-k_{2}$ ) where the singularity is at $T_{s}=1.22$.

A summary of the different regimes and the corresponding flow characteristics is shown in Table [1.

\begin{tabular}{||c|c|c||}
\hline Regime & Forward & Backward \\
\hline \hline$k_{1}>4 k_{2}$ & ancient & immortal \\
$\alpha, \beta, \gamma \neq 0$ & & \\
\hline$k_{1}<k_{2}$ & immortal & ancient \\
$\alpha, \beta, \gamma \neq 0$ & & \\
\hline$k_{2}<k_{1}<4 k_{2}$ & immortal & ancient \\
$\alpha, \beta, \gamma \neq 0$ & & \\
\hline$k_{1}<k_{2}$ & ancient & immortal \\
$\alpha=0, \beta, \gamma \neq 0$ & & \\
\hline$k_{1}<k_{2}$ & ancient & immortal \\
$\beta=0, \alpha, \gamma \neq 0$ & & \\
\hline \hline
\end{tabular}

TABLE I: The various cases in Bach flow of asymmetrically warped product manifolds

\section{F. Comparison with Ricci Flow}

We can easily compare the above results with those for un-normalized and normalised Ricci flows. For our asymmetrically warped metric, the un-normalised and normalised Ricci flow equations are, respectively,

$$
\frac{d A^{2}}{d t}= \pm 2 \frac{A^{2}}{C^{2}} k_{1}\left(k_{1}+2 k_{2}\right) \quad ; \quad \frac{d B^{2}}{d t}= \pm 2 \frac{B^{2}}{C^{2}} k_{2}\left(k_{1}+2 k_{2}\right) \quad ; \quad \frac{d C^{2}}{d t}= \pm 2\left(k_{1}^{2}+2 k_{2}^{2}\right)
$$



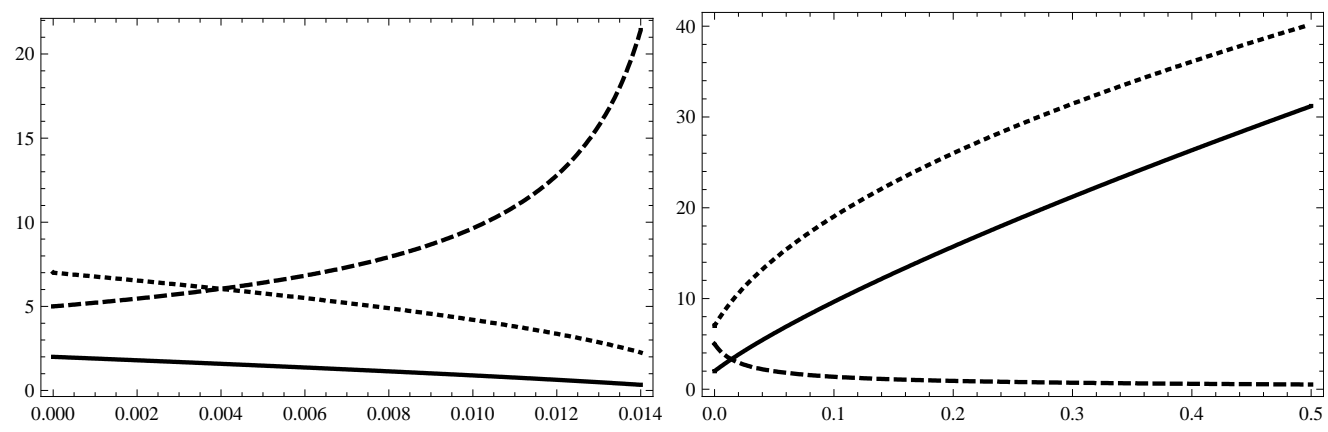

(a) $A^{2}(t)($ continuous $), B^{2}(t)($ dashed $), C^{2}(t)($ dotted $) \mathrm{v} / \mathrm{s} t$ for $k_{1}>4 k_{2}$
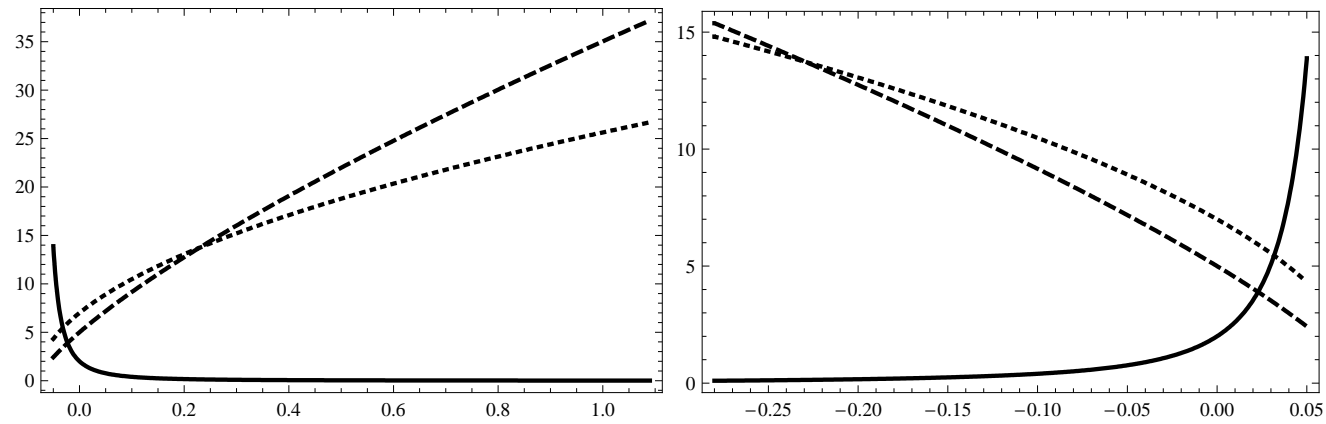

(b) $A^{2}(t)\left(\right.$ continuous), $B^{2}(t)($ dashed $), C^{2}(t)($ dotted $) \mathrm{v} / \mathrm{s} t$ for $k_{1}<k_{2}$
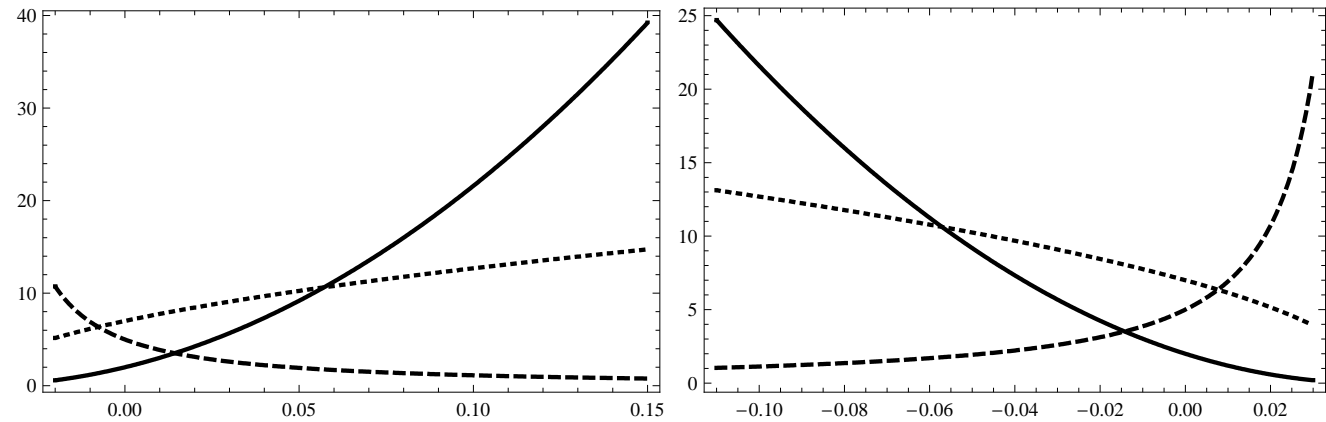

(c) $A^{2}(t)$ (continuous), $B^{2}(t)($ dashed $), C^{2}(t)($ dotted $) \mathrm{v} / \mathrm{s} t$ for $k_{2}<k_{1}<4 k_{2}$

FIG. 4: Bach flow : Forward and backward flow for different values of $k_{1}$ and $k_{2}$
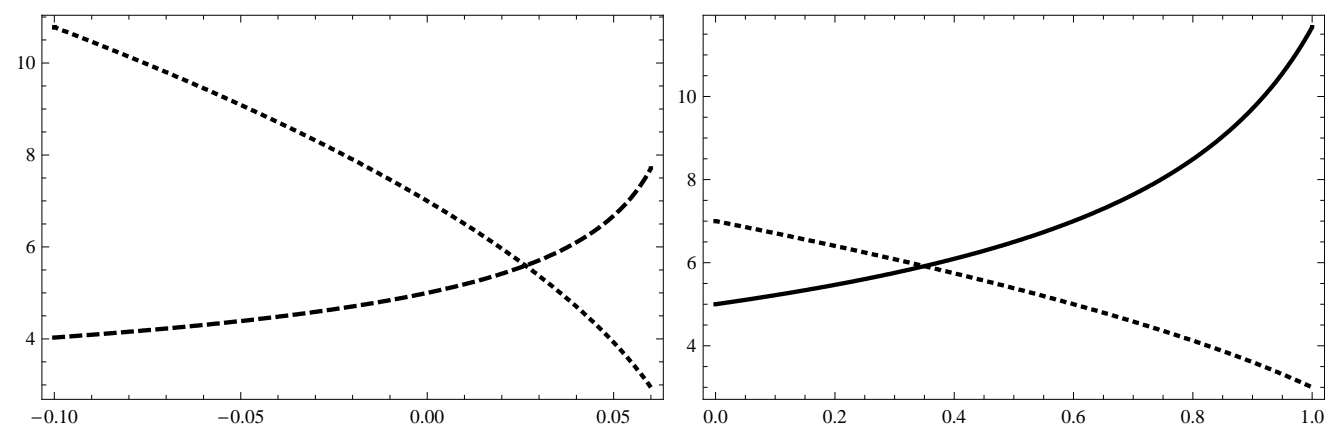

(a) $A^{2}(t)($ continuous $), B^{2}(t)($ dashed $), C^{2}(t)($ dotted $) \mathrm{v} / \mathrm{s} t$ for $k_{1}<4 k_{2}$

FIG. 5: Bach flow : For $\alpha=0$ (left ) and $\beta=0$ (right) respectively 


$$
\frac{d A^{2}}{d t}= \pm \frac{A^{2}}{C^{2}}\left(k_{1}-k_{2}\right)\left(k_{1}+3 k_{2}\right) ; \frac{d B^{2}}{d t}=\mp \frac{B^{2}}{C^{2}}\left(k_{1}-k_{2}\right)\left(k_{1}+k_{2}\right) ; \frac{d C^{2}}{d t}= \pm\left(k_{1}-k_{2}\right)^{2}(68)
$$

The solutions are straight forward and given below-the first set is for un-normalised flows and the next one is for normalised Ricci flow.

$$
\begin{gathered}
A^{2}(t)=A_{0}^{2}\left(C_{0}^{2} \pm 2\left(k_{1}^{2}+2 k_{2}^{2}\right) t\right)^{k_{1}\left(k_{1}+2 k_{2}\right) /\left(k_{1}^{2}+2 k_{2}^{2}\right)} \\
B^{2}(t)=B_{0}^{2}\left(C_{0}^{2} \pm 2\left(k_{1}^{2}+2 k_{2}^{2}\right) t\right)^{k_{2}\left(k_{1}+2 k_{2}\right) /\left(k_{1}^{2}+2 k_{2}^{2}\right)} \\
C^{2}(t)=C_{0}^{2} \pm 2\left(k_{1}^{2}+2 k_{2}^{2}\right) t \\
A^{2}(t)=A_{0}^{2}\left(C_{0}^{2} \pm 2\left(k_{1}-k_{2}\right)^{2} t\right)^{\left(k_{1}+3 k_{2}\right) /\left(k_{1}-k_{2}\right)}=A_{0}^{2}\left(C_{0}^{2} \pm 2\left(k_{1}-k_{2}\right)^{2} t\right)^{\frac{\alpha}{\gamma}} \\
B^{2}(t)=B_{0}^{2}\left(C_{0}^{2} \pm 2\left(k_{1}-k_{2}\right)^{2} t\right)^{-\left(k_{1}+k_{2}\right) /\left(k_{1}-k_{2}\right)}=B_{0}^{2}\left(C_{0}^{2} \pm 2\left(k_{1}-k_{2}\right)^{2} t\right)^{-\frac{\beta}{\gamma}} \\
C^{2}(t)=C_{0}^{2} \pm\left(k_{1}-k_{2}\right)^{2} t
\end{gathered}
$$

In the un-normalized Ricci flow, if $k_{1}=-2 k_{2}$ then $A^{2}(t)$ and $B^{2}(t)$ have no evolution while $C^{2}(t)$ grows linearly (the linear behaviour of $C^{2}(t)$ is a point of difference with the corresponding result for Bach flows). For normalized Ricci flow, $k_{1}=k_{2}$ implies that the R. H. S. of the flow equations are all zero and there is no evolution. Further, for normalized Ricci flow we note that the linear solution for $C^{2}(t)$ is similar to that for un-normalised flow, modulo a difference in the slope. However, depending on whether $k_{1}>k_{2}$ or $k_{1}<k_{2}$, the solutions for $A^{2}(t)$ and $B^{2}(t)$ have mutually opposite behaviour for normalised flows, though this is not the case for the unnormalised flow. We have plotted some of the abovementioned features, in the next figure, for forward Ricci flow. Here we have used the same (as for Bach flow) initial conditions, i.e. $\left(A_{0}^{2}, B_{0}^{2}, C_{0}^{2}\right)=(2,5,7)$ and $\left(k_{1}, k_{2}\right)=(8,1)$ for both unnormalized and normalized flows. The figure on the left which is for unnormalized Ricci flow, depicts the increasing nature of all the scale factors though $B^{2}(t)$ asymptotically reaches a constant value. This is an immortal flow with a singularity time $T_{s}=-0.053$. If we consider normalised flows, the immortal behavior $\left(T_{s}=-0.143\right)$ is retained, though all the scale factors are not increasing. $A^{2}(t)$ and $C^{2}(t)$ cross each other at some $t$ and diverge subsequently. On the other hand, $B^{2}(t)$ decays and asymptotically reaches a constant value. $B^{2}(t)$ also crosses $A^{2}(t)$ and $C^{2}(t)$ (not shown in the picture). The figure on the right shows 
the abovementioned behaviour for normalised Ricci flow.

The evolution of $A^{2}(t), B^{2}(t)$ and $C^{2}(t)$ for normalised Ricci flow turns out to be in powers of $t$ which are the squares of those for Bach flow. This is evident in the solutions$A^{2}(t)$ goes as $t^{\frac{\alpha}{\gamma}}$ for normalised Ricci while it varies as $t^{\frac{\alpha}{2 \gamma}}$ for Bach and so on. This is expected primarily because of the higher order nature of the Bach tensor.

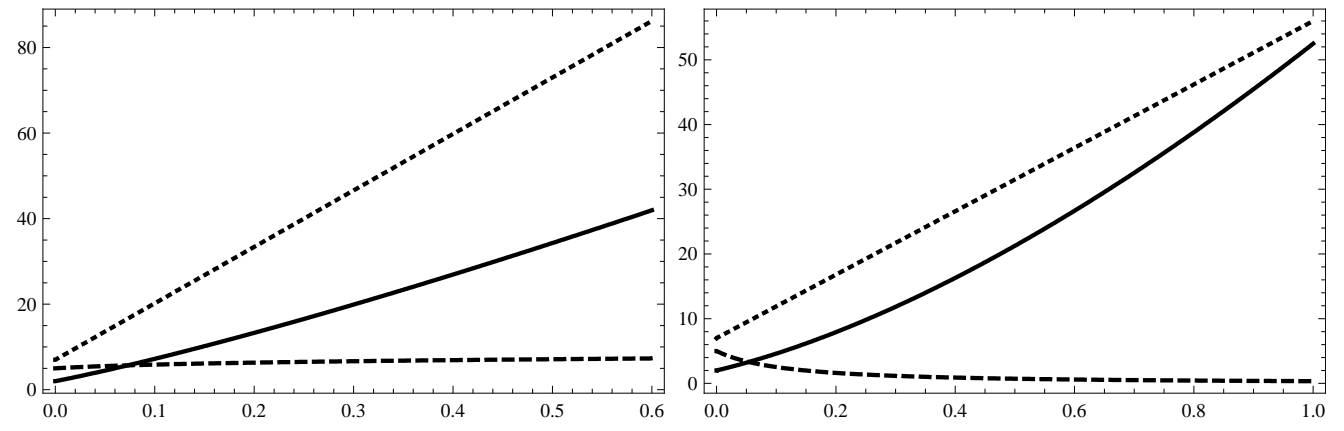

(a) $A^{2}(t)($ continuous $), B^{2}(t)($ dashed $), C^{2}(t)($ dotted $) \mathrm{v} / \mathrm{s} t$

FIG. 6: Ricci flow : unnormalized (left) and normalized (right)

\section{ASYMMETRICALLY WARPED PRODUCTS: GENERALIZED CASE}

In this section we briefly re-do our previous analysis for a more general case. Let us assume the line element as

$$
d s^{2}=-A^{2}(t) e^{2 f(\sigma)} d \tau^{2}+B^{2}(t) e^{2 g(\sigma)} d x^{2}+C^{2}(t) e^{2 h(\sigma)} d y^{2}+D^{2}(t) d \sigma^{2}
$$

let us assume further

$$
f(\sigma)=k_{1} \sigma \quad ; \quad g(\sigma)=k_{2} \sigma ; \quad h(\sigma)=k_{3} \sigma
$$


The Bach tensor components for the above mentioned line element are,

$$
\begin{aligned}
B_{t t} & =-\frac{A^{2}}{3 D^{4}} \alpha^{\prime} e^{2 \sigma \kappa_{1}} \\
B_{x x} & =-\frac{B^{2}}{3 D^{4}} \beta^{\prime} e^{2 \sigma \kappa_{2}} \\
B_{y y} & =-\frac{C^{2}}{3 D^{4}} \gamma^{\prime} e^{2 \sigma \kappa_{2}} \\
B_{z z} & =\frac{1}{3 D^{2}} \delta^{\prime} e^{-2 \sigma \kappa_{3}}
\end{aligned}
$$

where $\alpha^{\prime}, \beta^{\prime}$ and $\gamma^{\prime}$ are given as,

$$
\begin{aligned}
& \alpha^{\prime}=\left(\kappa_{1}^{2}+\left(\kappa_{2}-\kappa_{3}\right)^{2}-2 \kappa_{1}\left(\kappa_{2}+\kappa_{3}\right)\right)\left(\kappa_{1}^{2}-\kappa_{2}^{2}-\kappa_{2} \kappa_{3}-\kappa_{3}^{2}+\kappa_{1}\left(\kappa_{2}+\kappa_{3}\right)\right) \\
& \beta^{\prime}=\left(\kappa_{1}^{2}-\kappa_{2}^{2}-\kappa_{2} \kappa_{3}+\kappa_{3}^{2}+\kappa_{1}\left(\kappa_{3}-\kappa_{2}\right)\right)\left(\kappa_{1}^{2}+\left(\kappa_{2}-\kappa_{3}\right)^{2}-2 \kappa_{1}\left(\kappa_{2}+\kappa_{3}\right)\right) \\
& \gamma^{\prime}=\left(\kappa_{1}^{2}+\kappa_{1} \kappa_{2}+\kappa_{2}^{2}-\left(\kappa_{1}+\kappa_{2}\right) \kappa_{3}-\kappa_{3}^{2}\right)\left(\kappa_{1}^{2}+\left(\kappa_{2}-\kappa_{3}\right)^{2}-2 \kappa_{1}\left(\kappa_{2}+\kappa_{3}\right)\right) \\
& \delta^{\prime}=\left(\kappa_{1}^{2}+\left(\kappa_{2}-\kappa_{3}\right)^{2}-2 \kappa_{1}\left(\kappa_{2}+\kappa_{3}\right)\right)\left(\kappa_{1}^{2}+\kappa_{2}^{2}-\kappa_{2} \kappa_{3}+\kappa_{3}^{2}-\kappa_{1}\left(\kappa_{2}+\kappa_{3}\right)\right)
\end{aligned}
$$

The tracelessness condition becomes $\alpha^{\prime}-\beta^{\prime}-\gamma^{\prime}+\delta^{\prime}=0$.

If we put $k_{3}=0$ and $B(t)=C(t)$ then the Bach tensor components match with those mentioned in the previous section. The generic structure of the solutions will not be very different from the previous case and we do not discuss them any further here.

\section{REMARKS AND CONCLUSIONS}

We now list our conclusions briefly and make some concluding remarks.

We have solved the Bach flow equations on $(2,2)$ unwarped product manifolds such as $S^{2} \times S^{2}, R^{2} \times S^{2}$, by making use of the splitting of the Bach tensor for such manifolds. The flows conform to the traceless property of the Bach tensor and are quite different from Ricci flows on similar manifolds.

Further, we obtain the fixed point equations for the $(2,2)$ unwarped product manifolds and have also solved them for restricted cases. The metric functions and the scalar curvature are found analytically.

Finally, we look at warped product manifolds with a Lorentzian signature. For a special class of metric functions we reduce the Bach flow equations to a dynamical system and 
solve them explicitly. The evolution patterns of the various flow-parameter dependent scale factors are obtained and illustrated in detail. Here too the traceless and higher order characteristics of the Bach tensor play a role in distinguishing the evolution patterns from those for Ricci flows. We have compared the Bach flow solutions with those for Ricci flow and pointed out the differences.

It may be mentioned, that the solutions to the flow equations which we have obtained in the $(2,2)$ unwarped product manifold cases are also solutions of $4+1$ dimensional HoravaLifshitz gravity (for the restricted choice of the action, as mentioned in the Introduction). Another interesting offshoot of our calculations for warped manifolds is the Bach flat solution with $k_{1}=4 k_{2}$ given as (without the scale factors):

$$
d s^{2}=-e^{8 k_{2} \sigma} d \tau^{2}+e^{2 k_{2} \sigma}\left(d x^{2}+d y^{2}\right)+d \sigma^{2}
$$

This would be a non-singular vacuum solution in conformal gravity though we are not sure whether it is a new solution or a known solution written using un-conventional coordinates.

For future work, it may be worthwhile to find out examples where the higher derivative terms in the Bach tensor play an explicit role in determining the flow characteristics. This is a difficult task considering the fact that evaluating the Bach tensor is, in itself, quite complicated. Further, one may consider looking at generalisations of the Bach tensor in other dimensions [2] and define newer geometric flows in dimensions greater than four (note for three dimensions we have the Cotton flow which has been studied [19]). As mentioned before, like other geometric flows the Bach flow is derivable from an action principle (the Weyl-squared action). It may therefore be possible (and useful) to look into an entropy formula and associated geometric aspects [10]. We hope to return to these issues later.

[1] R. Bach, On Weyl's relativity theory and the Weylsian expansion of curved area concepts., Math. Ziets. 9, 110 (1921).

[2] J. Bergman, Conformal Einstein spaces and Bach tensor generalizations in $n$ dimensions, Ph.d thesis, Matematiska institutionen Linkopings universitet, Sweden (2004).

[3] L. Landau and E. M. Lifshitz, The Classical Theory of Fields, Pergamom Press, UK (1975).

[4] Some authors [2, 12, 13] use a different sign convention for which the Riemann tensor has a 
sign opposite to what we use here. The difference in sign shows up in the contracted Hessian of the Ricci tensor $\left(\nabla^{l} \nabla_{i} R_{l k}=\frac{1}{2} \nabla_{i} \nabla_{k} R-R_{i l} R_{k}^{l}+R_{i j k l} R^{j l}\right)$ and leads to a different definition of the Bach tensor given as $B_{i k}=\nabla^{j} \nabla^{l} C_{i j k l}-\frac{1}{2} R^{j l} C_{i j k l}$ (note the minus sign).

[5] I. Bakas, F. Bourliot, D. Lust and M. Petropoulos, Geometric flows in Horava-Lifshitz gravity, JHEP 2010, No. 4(2009)

[6] B. Chow and D. Knopf, The Ricci flow: an introduction, Mathematical Surveys and Monographs Vol. 110, AMS, Providence, 2004.

[7] D. Glickenstein Lectures on Ricci Flow Spring 2009, http://math.arizona.edu/ glickenstein/rf/

[8] R.S. Hamilton, Three-manifolds with positive Ricci curvature, J. Diff. Geom. 17, 255 (1982).

[9] D. Friedan, Nonlinear Models in 2+ $+\epsilon$ Dimensions, Annals of Physics 163, 318 (1985).

[10] G. Perelman, The entropy formula for the Ricci flow and its geometric applications, Preprint math.DG/0211159

[11] E. Bahuaud and D. Helliwell, Short time existence for some higher order geometric flows, Preprint math.10104287v1

[12] P. D. Mannheim and D. Kazanas, Newtonian Limit of Conformal Gravity and the Lack of Necessity of the Second Order Poisson Equation, Gen. Rel. and Grav. 26, No.4(1994)

[13] P. D. Mannheim, Alternatives to Dark Matter and Dark Energy, Prog. Part. and Nucl. Phys.56, No. 2(2006)

[14] B. Fiedler and R. Schimming, Exact solutions of the Bach field equations of general relativity, Rep. Math. Phys. 17, 15 (1980).

[15] K. Yano, Differential geometry on complex and almost complex spaces, Pergamon,. New York, 1965.

[16] S. Das, K. Prabhu, and S. Kar, Ricci flow of unwarped and warped product manifolds, Int. Jr. Geom. Meth. Mod. Phys. 7, 837 (2010)

[17] This was pointed out to us by G. Date.

[18] L. Randall and R. Sundrum, An alternative to compactification, Phys.Rev.Lett.83,4690 (1999) ibid. A large mass hierarchy from a small extra dimension, Phys.Rev.Lett. 83, 3370 (1999)

[19] A. U. O. Kisisel, O. Sarioglu, B. Tekin, Cotton flow, Class.Quant.Grav. 25, 165019 (2008). 